\title{
Desafios e potencialidades da interprofissionalidade no contexto do programa de educação pelo trabalho para saúde
}

Challenges and potentialities of interprofissionality in the contexto of the education through for health program

Retos y potencialidades de la interptofesionalidad em el contexto del program de educación para el trabajo em salud Universidade do Estado do Pará, Brasil

E-mail: lyviaoliveeira@gmail.com

Marwim Luis Batista Costa

ORCID: https://orcid.org/0000-0002-3787-1761 Universidade do Estado do Pará, Brasil E-mail: marwimlbcosta@hotmail.com

Nathália Cantuária Rodrigues

ORCID: https://orcid.org/0000-0001-9672-7113 Universidade do Estado do Pará, Brasil

E-mail: naathcr@gmail.com

Carla Miyuki Morita

ORCID: https://orcid.org/0000-0002-2821-7346 Universidade do Estado do Pará, Brasil

E-mail: carlamorita16@gmail.com

Maria Paloma Miranda Pereira

ORCID: https://orcid.org/0000-0002-4480-0777 Universidade do Estado do Pará, Brasil E-mail: maria.paloma18@gmail.com Monaliza dos Santos Pessoa

ORCID: https://orcid.org/0000-0002-4163-8081 Universidade do Estado do Pará, Brasil

E-mail: monapessoa@gmail.com

Erica Feio Carneiro Nunes

ORCID: https://orcid.org/0000-0002-1274-4686 Universidade do Estado do Pará, Brasil E-mail: ericacarneiro@uepa.br

\begin{abstract}
Resumo
Objetivo: relatar as dificuldades e os benefícios constatados em uma atuação interprofissional numa Unidade Básica de Saúde (UBS) localizada em um bairro periférico da capital paraense. Metodologia: trata-se de um estudo de caráter descritivo com abordagem qualitativa do tipo relato de experiência o qual apresenta atividades realizadas por estudantes de enfermagem e medicina em uma UBS no período de abril de 2019 a março de 2020. Neste período foram desenvolvidas atividades de consultas interprofissionais aos usuários, palestras e dinâmicas de educação em saúde e de educação continuada. Resultados: durante a participação, notaram-se desafios para a adoção das práticas interprofissionais, como a resistência dos profissionais de saúde e falta de espaço físico para as práticas. Entre os benefícios destas práticas, destaca-se a melhora no processo de construção do conhecimento e a elevação na qualidade dos cuidados prestados à comunidade. Conclusão: desta forma, conclui-se que o Programa de Educação pelo Trabalho
\end{abstract}


para Saúde é peça fundamental para a alteração do cenário para um contexto interprofissional, consolidando o conhecimento de estudantes e profissionais por meio da prática colaborativa na atuação multiprofissional.

Palavras-chave: Educação interprofissional; Estratégia saúde da família; Educação em saúde; Educação continuada; Ensino.

\begin{abstract}
Objective: to report the difficulties and benefits found in an interprofessional activity in a Basic Health Unit (UBS) located in a peripheral neighborhood of the capital of Pará. Methodology: this is a descriptive study with a qualitative approach, such as an experience report, which presents activities carried out by nursing and medical students in a UBS from April 2019 to March 2020. During this period, research activities were developed. interprofessional consultations with users, lectures and dynamics of health education and continuing education. Results: during participation, challenges were noted for the adoption of interprofessional practices, such as the resistance of health professionals and lack of physical space for practices. Among the benefits of these practices, there is an improvement in the process of building knowledge and an increase in the quality of care provided to the community. Conclusion: in this way, it is concluded that the Education through Work for Health Program is fundamental to change the scenario for an interprofessional context, consolidating the knowledge of students and professionals through collaborative practice in multiprofessional performance.
\end{abstract}

Keywords: Interprofessional education; Family health strategy; Health education; Education continuing; Teaching.

\title{
Resumen
}

Objetivo: reportar las dificultades y beneficios encontrados en una actividad interprofesional en una Unidad Básica de Salud (UBS) ubicada en un barrio periférico de la capital de Pará. Metodología: se trata de un estudio descriptivo con enfoque cualitativo, como un relato de experiencia, que presenta las actividades realizadas por estudiantes de enfermería y medicina en una UBS desde abril de 2019 a marzo de 2020. Durante este período, se desarrollaron actividades de investigación. Consultas interprofesionales con usuarios, charlas y dinámicas de educación en salud y educación continua. Resultados: durante la participación, se observaron desafíos para la adopción de prácticas interprofesionales, como la resistencia de los profesionales de la salud y la falta de espacio físico para las prácticas. Entre los beneficios de estas prácticas, se encuentra una mejora en el proceso de construcción de conocimiento y un aumento en la calidad de la atención brindada a la comunidad. Conclusión: de esta manera, se concluye que el Programa Educación a través del Trabajo para la Salud es parte fundamental para cambiar el escenario a un contexto interprofesional, consolidando el conocimiento de estudiantes y profesionales a través de la práctica colaborativa en el desempeño multiprofesional.

Palabras clave: Educación interprofesional; Estrategia de salud familiar; Educación en salud; Educación continua; Enseñanza.

\section{Introdução}

O movimento histórico de produção do conhecimento e as condições de vida e saúde da população foram repensados após a revolução industrial. Desde a década de 1970 preocupa-se em compreender os fatores responsáveis pelo processo saúdedoença. Autores e cientistas sociais propuseram modelos de aprendizagem para compreensão dos determinantes de saúde, sendo o mais aceito atualmente no Brasil o Modelo de Determinantes Sociais de Dahlgren e Whitehead, o qual estabelece os fatores que determinam o processo saúde-doença em diversos níveis, indo do individual ao macro social (Ceballos, 2015).

A partir de tal modelo, a saúde individual é influenciada primeiramente por fatores relacionados a idade, sexo, gênero e genética. Em uma segunda camada, encontra-se o estilo de vida e as escolhas pessoais. Na camada subjacente é possível observar as redes sociais e comunitárias juntamente com as condições de vida e de trabalho. E por fim, na última camada, identificam-se os macrodeterminantes, ou seja, condições socioeconômicas, culturais e ambientais em geral (Souza, 2017).

Diante da pluralidade de demandas, especificidades e constantes transformações do meio, encontram-se no diálogo, entre os diferentes campos de conhecimentos, ferramentas para abordar integralmente a complexidade dos problemas de saúde atuais, sendo o interprofissionalismo um dos seus pilares. O interprofissionalismo diz respeito a comunicação entre profissionais de diferentes áreas para a integração de um cuidado especializado, intencionalmente estabelecendo nexos e vínculos entre si para alcançar conhecimentos e decisões mais abrangentes diante de um mesmo objeto de trabalho, opondo-se ao reducionismo e a fragmentação (Freeth, 2007 apud Santana \& Rossit, 2017).

$\mathrm{Na}$ mesma conjuntura, destaca-se a interdisciplinaridade acadêmica como meio para facilitar práticas interprofissionais na área da saúde, de modo que o saber interdisciplinar dá condições ao profissional para perceber o homem como um todo com 
uma visão mais ampla, a qual ultrapassa as barreiras de cada área, levando ao fim da hegemonia de alguns saberes sobre os outros e à compressão das implicações sociais da sua prática (Farias et al., 2018).

Desse modo, com o objetivo de fomentar a formação profissional para melhor atender a população, o Ministério da Saúde institucionalizou o Programa de Educação pelo Trabalho para a Saúde (PET-Saúde), o qual articulado com os profissionais da Atenção Básica facilita o processo de integração do eixo ensino-serviço-comunidade, por meio da inserção de estudantes da área da saúde em Unidades Básicas de Saúde (UBS), norteados pela conceituação de interprofissionalismo, para realizar ações voltadas para o ensino, a pesquisa, a extensão universitária e a participação social (Brasil, 2018).

O programa objetiva possibilitar que o Ministério da Saúde cumpra seu papel de ordenador da formação de profissionais de saúde, além de contribuir para a implementação das Diretrizes Curriculares Nacionais dos cursos de graduação da área da saúde (Brasil, 2010). O PET-Saúde é voltado às instituições de educação superior públicas e privadas, possuindo como integrantes os estudantes regulamentes matriculados nas instituições, os tutores acadêmicos (docentes das instituições que orientem as vivências em serviço) e os preceptores (profissionais dos serviços de saúde que orientam os estudantes participantes do programa) (Brasil, 2010).

A inclusão do programa em UBS corrobora tanto para a qualificação dos profissionais atuantes na área quanto para o enriquecimento da formação acadêmica, tendo como principal beneficiário das ações a comunidade e famílias presentes (Lourenço et al., 2017). Nesse sentido, a Universidade do Estado do Pará (UEPA) em parceria com a Secretaria Municipal de Saúde de Belém desenvolveu ações e projetos vinculados ao PET-Saúde.

O presente trabalho tem como objetivo principal relatar as dificuldades e os benefícios constatados em uma atuação interprofissional numa Unidade Básica de Saúde (UBS) localizada em um bairro periférico na capital paraense. Ademais, busca conceituar a atuação interprofissional em saúde, discutir os fatores que influenciam na sua implementação e analisar o contexto da saúde no Brasil.

\section{Metodologia}

Trata-se de um estudo do tipo relato de experiência de caráter descritivo com abordagem qualitativa, o qual é caracterizado pela facilidade de explorar as consequências das intervenções realizadas, pois este tipo de metodologia é aberto a receber as informações e demandas que surgem e não apenas sugestões pré-definidas. Na área da saúde tais estudos são fundamentais para analisar o contexto e as percepções de uma determinada situação, possibilitando a evidência do impacto das intervenções (Souza et al., 2018). As atividades foram realizadas por estudantes da área da saúde no período de abril de 2019 a março de 2020, contendo uma carga horária semanal de oito horas em uma UBS.

O PET-Saúde encontra-se alojado na UEPA, abrangendo os cursos de medicina, enfermagem, fisioterapia, terapia ocupacional, educação física e biomedicina. Dentre os participantes, obteve-se a divisão em grupos, formados por um coordenador-tutor, tutores, preceptores e estudantes. Os últimos foram divididos em grupos menores de acordo com o preceptor denominado, atuando nos serviços alocados.

O presente trabalho destaca a experiência do grupo alocado na UBS São Joaquim, contando com a participação de estudantes de enfermagem e medicina durante a realização das atividades propostas pelo PET-Saúde. As atividades do programa iniciaram com um processo de reconhecimento da área abrangida pela UBS, juntamente com o levantamento do perfil dos usuários da comunidade atendida, sendo observados os principais problemas de saúde enfrentados por estes. Durante este processo foi necessário o auxílio dos Agentes Comunitários de Saúde (ACS), os quais relataram, também, as dificuldades vivenciadas durante cumprimento de suas funções. 
De acordo com as demandas apresentadas pelos funcionários e usuários, o grupo formulou um cronograma de atividades abrangendo os 11 meses do programa, as quais se dividiram em: 1) atendimento interprofissional; 2) atividades de educação em saúde com os usuários; 3) atividades de educação continuada.

\section{Atendimento interprofissional}

Os atendimentos eram realizados semanalmente de acordo com a marcação das consultas médicas dos usuários. Em um primeiro momento era realizada a consulta da equipe de enfermagem seguida pela consulta com a equipe médica. Os estudantes eram responsáveis pelo o atendimento, sendo observados e auxiliados pelos preceptores, especialmente no momento da realização da conduta.

Entre o serviço da equipe de enfermagem e o da equipe médica, os estudantes eram responsáveis por repassar o caso de uma equipe à outra e, após a realização das consultas, as duas equipes elaboravam em conjunto a formulação da conduta do paciente. As condutas abrangiam desde um simples encaminhamento a um serviço mais especializado a um plano terapêutico anual de cuidado acompanhado.

Mensalmente eram realizadas as consultas da equipe do Núcleo de Apoio à Saúde da Família (NASF), compostas por profissionais da nutrição, psicologia, terapeuta ocupacional e fisioterapeuta. Os estudantes por vezes acompanhavam este atendimento e auxiliavam na discussão dos casos abordados. Ao final realizava-se análises interprofissionais sobre os casos clínicos que deveriam ser entregues ao preceptor.

\section{Atividades de educação em saúde}

As atividades de educação em saúde ocorriam de duas a quatro vezes por mês e basearam-se em palestras voltadas aos usuários que se encontravam em sala de espera para as consultas. As temáticas das atividades eram formuladas de acordo com o calendário do ministério da saúde.

À priori, os estudantes reuniam-se com a preceptora para organizar a abordagem que seria realizada, formulando cartazes e folders para ilustração dos temas. Nas ações, inicialmente os estudantes questionavam os pacientes afim de entender o grau de conhecimento deles sobre o assunto para que então pudessem realizar a correção ou soma deste sobre o tema. As temáticas abordadas incluíam Hipertensão Arterial, Diabete Mellitus, saúde mental, prevenção ao câncer de mama, combate à obesidade, combate ao fumo, prevenção ao câncer de próstata e a luta contra a AIDS (Síndrome da Imunodeficiência Humana Adquirida).

\section{Atividades de educação continuada}

As atividades de educação continuada eram voltadas para os ACS da UBS, ocorrendo uma vez por semana pela manhã. Em um primeiro momento, os estudantes realizaram uma discussão com os ACS para compreender as principais demandas da área e dificuldades que estes profissionais encontravam no exercício da sua função. Diante disso, os estudantes estabeleceram as temáticas pertinentes e iniciaram atividades voltadas para a discussão de casos clínicos, dinâmicas e debates temáticos com temas selecionados pelos ACS.

\section{Resultados e Discussão}

Durante os períodos das práticas relatadas, era notória a importância da interprofissionalidade no contexto da atenção básica, visto que está de íntimo acordo com o princípio da longitudinalidade do Sistema Único de Saúde. A interprofissionalidade resulta da necessidade de compreender o processo saúde-doença a partir de um conceito ampliado de saúde, uma vez que este processo é compreendido como produto da interação de várias dimensões, como a biológica, a psicológica, a econômica, a 
cultural, a social, a individual e a coletiva (Silva; Bastitella \& Gomes, 2007; Grillo; Ibarra \& Mezadri, 2019). Esses determinantes deixam em evidência a complexidade inerente ao campo da saúde, exigindo a participação colaborativa de diferentes profissionais de forma a construir conhecimentos com base em disciplinas distintas, em um processo interdisciplinar e interprofissional (Rios; Sousa \& Caputo, 2019).

A prática comunicativa entre a equipe se torna responsável por diminuir a demora no atendimento pela busca de informações já obtidas na consulta anterior, visto que, apesar de abrangerem ciências distintas, ambas apresentam o mesmo objeto de trabalho (Araújo, 2014). Desse modo, ao contar com a contribuição de cada área no processo da formação de saúde integral do indivíduo, caracteriza-se uma atuação multidisciplinar sob ótica interprofissional.

O cuidado integral à saúde exige um elenco variado de profissionais, a fim de contemplar as diversas dimensões presentes nas demandas de saúde do usuário, família e comunidade. Nesse sentido é possível observar a necessidade da colaboração e da comunicação entre os profissionais, de modo a proporcionar uma atenção centrada ao paciente, uma vez que deve buscar integrar ações de promoção, prevenção, recuperação e reabilitação da saúde deste usuário por meio do serviço em saúde fornecido pela equipe multiprofissional (Agreli; Peduzzi \& Silva, 2016).

A partir da vivência com a equipe do NASF, foi possível observar as dificuldades encontradas para sua efetivação, tais como a influência da estrutura física da Unidade Básica de Saúde em uma consulta qualificada, de modo que o número de consultórios disponíveis não era compatível com a quantidade de profissionais da saúde. Todavia, também se constatou a importância da sua atuação frente ao cenário da Atenção Básica em Saúde, uma vez que trabalha com atendimento compartilhado, discussão de casos clínicos e construção conjunta de projetos terapêuticos, o que se torna uma oportunidade de compreensão do funcionamento do trabalho em saúde e interprofissional. Inúmeras competências são necessárias para se trabalhar no campo da saúde, de modo que em diferentes momentos é imprescindível mobilizar conhecimentos, esquemas e vivencias previas para o desenvolvimento de respostas criativas e reflexivas para as situações do cotidiano (Rios; Souza \& Caputo, 2019).

Dentre as atividades de educação em saúde com os usuários, destaca-se a realização de quatro palestras, no mês de maio, acerca do programa HIPERDIA do Ministério da Saúde. Este programa tem por objetivo cadastrar e acompanhar todos os pacientes hipertensos e diabéticos a fim de que através do cuidado especial consiga-se fazer um controle das doenças e garantir uma melhor qualidade de vida aos pacientes. Durante o ciclo de palestras, os estudantes organizaram uma dinâmica em que se escreveu em diversos papéis alguns mitos e verdades sobre as duas doenças (Brasil, 2002). A partir disto, os pacientes retiravam um papel da caixa e comentavam se consideravam aquilo um mito ou uma verdade. Diante disto, os estudantes poderiam corrigir e informar corretamente os usuários sobre a informação contida no papel. Na última palestra sobre o tema, foi oferecido um "café da manhã saudável", utilizando disto para discutir sobre a importância de uma alimentação balanceada no controle do Diabetes mellitus e da Hipertensão Arterial Sistêmica. Ambas as metodologias utilizadas nas atividades se mostraram benéficas, visto que conseguiu eliminar dúvidas que os usuários ainda possuíam, mesmo que já tenham um longo tempo de doença.

Ademais, evidencia-se o ciclo de palestras em alusão o "setembro amarelo", campanha do Ministério da Saúde de prevenção ao suicídio. Realizou-se 3 palestras durante o mês de setembro de 2019 com as seguintes temáticas cada: 1) transtorno de ansiedade generalizada; 2) transtorno depressivo maior; e 3) suicídio. A metodologia utilizada consistia na identificação dos sintomas destes problemas de saúde e das principais maneiras de prevenção e tratamento. A abordagem relacionada a este tema permitiu que alguns usuários expusessem situações vivenciadas em casa ou no trabalho que colocaram sua saúde mental em risco, além de relatarem experiências vividas com outros membros da família, as quais traziam aflição para o âmbito familiar.

As atividades de educação em saúde estimularam a prática colaborativa entre os estudantes, desde o planejamento até a execução da atividade com o diálogo de saberes. Além disso, foi possível observar o desenvolvimento de um elo de confiança e reciprocidade entre os estudantes, os profissionais de saúde e a comunidade, sendo nítida a participação dos usuários ao trazer à discussão relatos próprios e de familiares próximos, o que proporcionou a troca de conhecimentos e de vivências durante as 
rodas de conversa. Logo, uma abordagem técnica e científica que é adequada ao entendimento do público-alvo auxilia tanto na prevenção primária quanto na redução de agravos decorrentes de patologias (Vasconcelos et al., 2017).

A respeito das temáticas educacionais abordadas com a comunidade, observou-se que cada estudante analisou os discursos e apresentou suas explicações de acordo com sua área de formação, de modo a favorecer o aprendizado mutuo e solucionar problemas a partir de proposições diferentes, permitindo a atuação interprofissional no enfrentamento das demandas em saúde em toda sua complexidade e desmistificando os assuntos abordados diante dos usuários (Rios; Sousa \& Caputo, 2019).

É necessário ressaltar que a formação interprofissional exige o desenvolvimento de competências em comum entre os cursos de graduação para alcançar uma assistência integral. Diante disso é fundamental possuir um aporte teórico fortalecido pelos cursos de graduação, respeitando as especificidades de cada profissão uma vez que a pratica colaborativa é essencial, assim como a escuta qualificada, a empatia, a utilização adequada da linguagem de acordo com o público e a capacidade de trabalhar em equipe e de analisar de forma ampla os problemas identificados (Rios; Sousa \& Caputo, 2019).

Entretanto, observa-se nas Instituições de Ensino Superior a valorização da formação uniprofissional e da efemeridade das ações de educação interprofissional, permanecendo como desafio a aplicação da interprofissionalidade na formação dos profissionais da saúde, visto que no início das atividades, os estudantes encontraram dificuldade para formular ações que abrangiam as áreas das saúdes de seus respectivos cursos, o que deveria ser estimulado pelas universidades. Isso é resultado da resistência dos docentes na flexibilização de seus domínios intelectuais, além do trabalho especializado realizado pelos próprios estudantes, favorecendo o pensamento hegemônico dicotômico e fragmentador dominante na sociedade (Rios; Sousa \& Caputo, 2019).

Do mesmo modo, faz-se de suma importância ressaltar o protagonismo da Atenção Básica à Saúde, a qual é a principal responsável pela reformulação e organização do sistema público de saúde e é regulamentada pela Política Nacional de Atenção Básica (PNAB) do Ministério da Saúde (Brasil, 2017). O desenvolvimento de políticas voltadas diretamente para o setor e a implementação de projetos, como a Estratégia Saúde da Família (ESF), impulsionaram a expansão da cobertura territorial da Atenção Básica, visto que ao longo dos anos constatou-se que quanto maior a cobertura da ESF menor o número de internações hospitalares da população local (Cecílio \& Reis, 2018).

Durante a realização das atividades planejadas com os ACS, foi possível encontrar empecilhos relacionados à estrutura física do local, como a falta de uma sala de reunião e equipamentos que efetivassem ergonomicamente a postura profissional, e relacionados a própria equipe de profissionais da saúde, que por vezes mostraram-se resistentes a ações de educação em saúde. Nas discussões de casos clínicos os ACS inferiam a conduta que consideravam correta para que então houvesse o debate. Além disso, relatavam situações semelhantes que já vivenciaram para contribuir com o caso de forma a pensar criticamente sobre sua realidade.

De acordo com a PNAB, todas as unidades de saúde devem oferecer um conjunto de infraestrutura e ambiência que são responsáveis pelo funcionamento da atenção básica, destacando-se que os parâmetros estruturais devem estar em consonância com a demandas da população atendida e com os processos de trabalho das equipes. A política considera ainda a implementação de espaços físicos adequados para a formação de estudantes e trabalhadores de saúde, proporcionando um ambiente de convivência interprofissional (Brasil, 2017).

Entretanto, o direcionamento de recursos para a qualificação estrutural e profissional da área ainda segue sob subfinanciamento per capita, influenciando na qualidade do serviço prestado e na efetividade do cumprimento de seus princípios e diretrizes. Outrossim, faz-se presente o desafio na produção de trabalhadores proativos e de gestores que realmente conheçam as políticas e propostas de intervenção, indo contra os modos tradicionais de se fazer gestão (Chioro et al., 2016 apud Cecílio \& Reis, 2018). 
Além disso, a ausência de capacitações/qualificações dificulta a atuação interprofissional e o direcionamento das atividades a serem propostas para a UBS, em consequência, promovendo a segmentação profissional e impondo barreiras para a construção de um atendimento integral ao usuário (Vendruscolo et al., 2020)

\section{Considerações Finais}

Conclui-se que as atividades realizadas nesse período foram de suma importância para a formação dos estudantes integrados no programa. A interprofissionalidade torna-se cada vez mais necessário no processo de aprendizagem na graduação da área da saúde e no processo de cuidado integral aos usuários. Destaca-se que a abordagem interprofissional promoveu um maior contato dos estudantes com os profissionais de saúde de diversas áreas, contribuindo para a construção do conhecimento. Ademais, a elaboração das atividades com a colaboração da equipe multiprofissional forneceu um arcabouço teórico importante para os estudantes participantes do PET-Saúde.

Com a experiência, obteve-se um maior contato da graduação com a Atenção Básica à Saúde, proporcionando aos estudantes melhores noções de gestão, do funcionamento da Rede de Atenção à Saúde e do processo saúde-doença, e como ele ocorre nos serviços de baixa complexidade do Sistema Único de Saúde. A abordagem com os ACS aproximou estes profissionais de um olhar mais cuidadoso em relação às suas necessidades e dificuldades no enfrentamento das atividades laborais, de modo que estes empecilhos foram explanados e escutados pelos estudantes e transmitidos à gestão da Unidade Básica de Saúde.

Os benefícios à população incluem o maior acesso a informações sobre temas importantes no contexto da saúde da comunidade. Ademais, a abordagem interprofissional nas consultas proporcionou um melhor cuidado com a saúde dos usuários.

Entretanto, notam-se ainda empecilhos na absorção das práticas interprofissionais, principalmente por parte dos profissionais de saúde atuantes, que frequentemente se recusam a adotar esta prática, devido à falta de qualificações e capacitações pertinentes sobre o assunto e pela ausência de aporte físico na ambiência local. Desta maneira, destaca-se a necessidade de ampliar o acesso aos trabalhadores da área da saúde as ações que estimulem a interprofissionalidade, principalmente aqueles alocados na atenção básica. A formulação de capacitações e dinâmicas sobre o tema, acrescido de enriquecimento do arcabouço científico que comprove a efetividade destas ações, têm papel fundamental neste processo.

\section{Agradecimentos}

Ao Ministério da Saúde e a Universidade do Estado do Pará (UEPA) pelo apoio aos estudantes bolsistas e voluntário aprovados no Edital 69/2018 Programa de Educação pelo Trabalho para Saúde (PET-Saúde/Interprofissionalidade) - UEPA, a Preceptora Monaliza dos Santos Pessoa pela condução da assistência interprofissional na UBS, e a Coordenadora do PETSaúde/Interprofissionalidade/UEPA Erica Feio Carneiro Nunes pela revisão do manuscrito durante sua elaboração e pelos direcionamentos das atividades promovidas a partir deste programa de extensão universitária, juntamente com a Tutora Sandra Suely de Veiga Baia.

\section{Referências}

Agreli, H. F., Peduzzi, M. \& Silva, M. C. (2016). Patient centred care in interprofessional collaborative practice. Interface, 20 (59), 905-916. http://dx.doi.org/10.1590/1807-57622015.0511.

Araújo, P. C. (2014). Trabalho em equipe multiprofissional na Estratégia Saúde da Família: a interface entre a equipe de Saúde Bucal e a equipe de Saúde da Família. Dissertação [Mestrado Profissional] Fiocruz. Escola Nacional dessaúde Pública Sérgio Arouca. http://dx.doi.org/10.1590/S1414-32832013005000006

Brasil. Ministério da Saúde (2018). Programa de educação pelo trabalho para a saúde (PET-Saúde). https://antigo.saude.gov.br/images/pdf/2018/dezembro/13/Programa-de-Educacao-pelo-Trabalho-para-a-Saude-SaudeGraduaSUS-FINAL-WEB.pdf

Brasil. Ministério da Saúde (2017). Portaria n².436, de 21 de setembro de 2017. Aprova a Política Nacional de Atenção Básica, estabelecendo a revisão de diretrizes para a organização da atenção básica no âmbito do Sistema Único de Saúde (SUS). Diário oficial da união. https://bvsms.saude.gov.br/bvs/saudelegis/gm/2017/prt2436_22_09_2017.html 
Research, Society and Development, v. 10, n. 4, e22010414041, 2021

(CC BY 4.0) | ISSN 2525-3409 | DOI: http://dx.doi.org/10.33448/rsd-v10i4.14041

Brasil. Ministério da Saúde (2010). Portaria interministerial no 421, de 3 de março de 2010. Institui o Programa de Educação Pelo Trabalho Para a Saúde (PETSaúde) e dá outras providências. Diário oficial da união. http://bvsms.saude.gov.br/bvs/saudelegis/gm/2010/pri0421_03_03_2010.html

Brasil. Ministério da Saúde (2002). Hiperdia - Sistema de Cadastramento e Acompanhamento de Hipertensos e Diabéticos. Manual de Operação. 2002. DATASUS. http://tabnet.datasus.gov.br/cgi/hiperdia/Cad_Acomp_Hipert_Diabet_2002.pdf

Cerballos, A. G. C. (2015). Determinação social do processo saúde e doença. In: Cerballos, A. G. C. Modelos conceituais de saúde, determinação social do processo saúde e doença, promoção da saúde. Recife: Grupo saber tecnologias educacionais e sociais UNA-SUS UFPE, 2015. https://ares.unasus.gov.br/acervo/html/ARES/3332/1/2mod_conc_saude_2016.pdf

Cecílio, L. C. O. \& Reis, A. A. O. (2018). Apontamentos sobre os desafios (ainda) atuais da atenção básica. Cadernos de saúde pública, 34 (8), 1-14. https://doi.org/10.1590/0102-311X00056917

Farias, D. N., Ribeiro, K. S. Q. S. \& Anjos, U. U. \& Brito, G. E. G. (2018). Interdisciplinaridade e interprofissionalidade na Estratégia Saúde da Família. Trab. Educ. Saúde, 16 (1), 141-162. http://dx.doi.org/10.1590/1981-7746-sol00098

Grillo, L. P., Ibarra, L. S. \& Mezadri, T. (2019). Conceito ampliado de saúde na formação dos profissionais de educação física. Saúde e Pesquisa, 12 (2), 265 273. https://doi.org/10.17765/2176-9206.2019v12n2p265-273

Lourenço, A. E. P., Cordeiro, A. A., Capeli, J. C. S., Oliveira, R. B. A., Pontes, P. V., Almeida, M. F. L. \& Barros, L. B. (2017). Programa de educação pelo trabalho para a saúde (PET-Saúde) e a formação do nutricionista num campus de interiorização. Demetra: alimentação, nutrição \& saúde, 12 (1), $41-58$. https://doi.org/10.12957/demetra.2017.26214

Rios, D. R. S., Sousa, D. A. B. \& Caputo, M. C. (2019). Diálogos interprofissionais e interdisciplinares na prática extensionista: o caminho para a inserção do conceito ampliado de saúde na formação acadêmica. Interface (Botucatu), 23. http://dx.doi.org/10.1590/interface.180080

Santana, M. C. C. P. \& Rossit, R. A. S. (2017). Interprofissionalismo nas residências multiprofissionais em saúde: análise na região nordeste do Brasil. Journal of management and primary health care, 8 (3), 45-46. https://doi.org/10.14295/jmphc.v8i3.609

Silva, J. P. V., Batistella, C. \& Gomes, M. L. (2007). Problemas, necessidades e situação de saúde: uma revisão de abordagens para a reflexão e ação da equipe de saúde da família. In: Fonseca, Angélica Ferreira, Corbo, Ana Maria D’Andrea (Org.). O território e o processo saúde-doença. Rio de Janeiro: EPSJV/FIOCRUZ, 2007. (Coleção Educação Profissional e Docência em saúde: a formação e o trabalho do agente comunitário de saúde, 1). 159-176. https://www.arca.fiocruz.br/handle/icict/39205

Souza, C. D. F. (2017). Determinantes sociais de saúde vs determinação social de saúde: uma aproximação conceitual. In: Webcongresso Internacional De Direito Sanitário, I, Anais eletrônicos. Cad. Ibero-Amer. Dir. Sanit., 116-124. https://doi.org/10.5935/0103-1104.20140082

Souza, D. L. B., Nunes, G. L. A., Bedaque, H. P. \& Carvalho, Y. M. Estudos Ecológicos. In: Bedaque, H. P., Bezerra, E. L. M. Descomplicando MBE: uma abordagem prática da medicina baseada em evidências. Caula de Papiro. 29-38. http://www.amape.com.br/wp-content/uploads/2019/04/LivroDescomplicando-MBE.pdf

Vasconcelos, M. I. O., Farias, Q. L. T., Nascimento, F. G., Cavalcante, A. S. P., Mira, Q. L. M., \& Queiroz, M. V. O. (2017). Educação em saúde na atenção básica: uma análise das ações com hipertensos. Rev. APS, 20 (2), 253-262. https://doi.org/10.34019/1809-8363.2017.v20.15943

Vendruscolo, C., Trindade, L. L., Maffissoni, A. L., Martini, J. G., Silva Filho, C. C. \& Sandri, J. V. A. (2020). Implicação do processo de formação e educação permanente para atuação interprofissional. Rev Bras Enferm, 73 (2). https://dx.doi.org/10.1590/0034-7167-2018-0359 